\title{
A New Method for Multi-hop Communication Over Bluetooth in Smartphones
}

\author{
A.Prakash $^{1}$, A.Pavithra ${ }^{2}$, M.Taneesh Reddy ${ }^{3}$ \\ ${ }^{1}$ Assistant Professor, KPRIT, Ghatkesar \\ ${ }^{2}$ Student, KPRIT, Ghatkesar \\ ${ }^{3}$ Student, KPRIT, Ghatkesar
}

\begin{abstract}
:
In this investigation, we built up a versatile application that gives scatternet organize setup utilizing Bluetooth interface on advanced mobile phones running Android Operating System to meet the communication needs of individuals, where there is no Central Access Point, the Internet or physical framework on the earth. The messages transmitted on the system are transmitted shared in a multi-hop way. Each gadget that joins the system can act both server and shopper jobs. Every gadget likewise fills in as a switch. Companions partaking in the system can take in the current condition of the system from the gadget to which they are connected as a tree structure. Along these lines, each associate takes in the current structure of the system. In the meantime, a calculation was created and executed to spread the data of the recently included gadget to all reporters the system. The created application design is planned to be utilized as an elective specialized strategy in a wide range of outside and indoor situations, in regions where normal conditions, for example, mines are hard to impart, cataclysmic event territories, protect tasks, digital fear monger assaults and running remote sensor systems.
\end{abstract}

Keywords: multi-hop networks; scatternet; Bluetooth; Android; ad- hoc networks

\section{Introduction}

Today, smart phones have a high capacity, a wide variety of hardware and a capacity to meet energy requirements. Communication technologies used in smart phones are Internet, GSM, NFC, WiFi and Bluetooth. This study focuses on Bluetooth and applications. Bluetooth was first updated in 1994 after being announced by Ericsson and by increasing its popularity and functionality, it became the standard in the market. When Bluetooth is available in version 4.0, the personal area is widely used in network applications. It is especially used in salk, fitness, home elence systems and computer peripherals.

Up to eight Bluetooth devices come together to form the alara piconet. Scatternet network with more than one piconet. The different piconets use different frequency-tabbed arrays to ensure that the interference is of low size [10]. Piconet alar is based on master / slave construction. According to the traditional a terminology, there is a topology. The device, which has a strategically central physical location, acts as a server. The surrounding devices assume the role of the consumer. In this study, the devices undertaken by the devices are subject to the standards of Bluetooth technology.

The Android API introduced by the Bluetooth API is developed in accordance with this terminology. Developers need to develop their own algorithms to create scatternet and implement their own design using the interfaces offered by the API.

In this study, we recommend selecting the Android platform to set up a scatternet with a network topology, updating the routing steps on devices for each device, setting up the network 
and updating the network back to the device. All devices are provided with the physical structure of the network to create the same in their own structure. With the developed demo messaging application, the recommended protocol is snapped. The application has been tested on 5 devices including 3 Samsung S3, 1 Samsung S4 and 1 Samsung S5. In contrast to the assumptions of our approach, a real application is developed and implemented on the scatternet network Android. Tests were performed in open and closed areas and results were observed. No simulation environment is preferred. In this study, multi-tab communication is performed in the application layer using Bluetooth's basic functions, unicast, multicast and broadcast communication methods.

In the remaining part of the article, there are studies about the scatternet and multi-tabular communication techniques in the literature. In the chapter 3, we describe the multi-tabbed messaging algorithm proposed in the article. Application analysis and evaluations are given in section 4 . In the last section, the application analysis and the results obtained are mentioned.

\section{II . RELATED WORK}

The main motivation for carrying out this study is to observe the feasibility of a functional communication method that can be used practically between smart phones and cellular phones, regardless of the cellular network and Internet. When the available technologies are examined, WiFi, WiFi Direct, NFC and Bluetooth technologies are encountered [1]. In terms of the Android Operating System, APIs are provided by Google that allow these technologies to communicate with the clans that can be used. Software developers are moderated with permissions and authorizations of APIs to manage the hardware.

The officially developed API for communicating between Android devices is a representation of the feature of the WiFi Direct protocol. WiFi Direct is a certified protocol for direct communication between Android devices with WiFi adapter. It is often used to share audio, images and documents between the phone and home electronics such as printers, cameras, televisions [2]. Different hardware manufacturers certify the devices they produce by providing the requirements of the WiFi Direct protocol. WiFi Direct installs a topology between devices, placing one device at a central location, and other devices are a member of the established group. Both the group constructor and the group member role cannot be assigned to a device as required by the protocol, and each device can only be a member of a group. This kst can be overwritten by having a rootsystem administrator role on the Android device. However, this is not a realistic approach considering real life practices. It is not possible for each user to perform the root process. Also, when $\mathrm{WiFi}$ Direct is examined in detail, the programming interfaces appear to have not matured sufficiently. WiFi circuits and hardware drivers produced by different hardware manufacturers are not compatible with each other. The Android-enabled e-communication scenario is possible with a small number of devices with a maximum number of tabs in a range [11].

NFC technology cannot be used to install. Data can be transferred between the NFC reader and the NFC tag at a distance of about $10 \mathrm{~cm}$. Considering real life scenarios, it is not possible to construct a multi-tabbed message transmission. Among the alternative technologies, Bluetooth offers the best available Bluetooth for Android.

In the literature, studies have been done to create a scatternet designed to provide single and multitabbed communication via Bluetooth. Studies are independent of the platform. The proposed methods and developed algorithms are not available on a real mobile platform. The findings of the surveys are mostly based on wireless detectors $[14,15,16]$. The studies done with Alglayc alar have been observed in the simulation environment and observed by the researchers. The most common ns-2 simulation environment is 
used. Thanks to the Ns-2, the proposed algorithms are on combo over 100 virtual coils.

There is a bass communication method which can be used for radio communication between the studies [3]. It is aimed to create a draft that will enable the common use of personal space and scatologies. The devices that remain in the cut-off area between the piconet are in the bridge position. With Broadcast, it is intended to spread the sound over the a tab. With the routing of the data packets in Scatternet, they are faced with many studies dealing with the subject in different aspects [4,5]. Location Aware Routing Protocol (LARP) is one of the recommended package routing methods. It is recommended that the minimum number of tabs and messages be transmitted over the network by accepting the physical locations of the Bluetooth devices and the distance between them.

The proposed algorithms for setting up scatternet contain differences according to the approaches they use. One of the algorithms prefers to centralize one of the devices [6]. When the messages are sent to the target device, a large number of devices require a tab operation. This method, which is not seen as efficient for dynamic fields, is not considered as an appropriate approach to growth in growth. For single-tab Bluetooth devices, all devices must be within range to reach each other with a single tab. However, this does not apply to very tabular alar $[7,8,9]$. Not all devices need to be in direct communication with each other in multi-tabbed locations. In this way, a larger communication area can be obtained.

The studies in the literature mainly focus on three subjects in the field of mobile space. The first category covers the process of forming the network. The second part consists in conducting the steering procedures and defining the driving paths. The last one is to maintain and maintain current routing paths. In our study, the traditionally accepted Bluetree algorithm was applied. It is preferred for its coverage with its simplicity and Bluetooth reporting on Android $[15,17]$. A proactive protocol was developed for island routing. The advantage over reactive algorithms, which is the alternative, is that when the data is sent between the two devices, the guidance path does not need to be asked to the surrounding commutations. Reagent protocols include Ad-hoc On Demand Distance Vector (AODV) and Dynamic Source Routing (DSR) protocols, which are useful in our research [18].

In some studies, it has benefited from helpful communication elements such as GPS and Internet. In general, the existing bases in the real mobile platforms are ignored. In the programmable interfaces of Bluetooth technology on smart-phones, it is not to be emphasized

that the user is able to determine the time of the interleaving time and the devices need to be pegged to simplify communication in the simplest sense. Considering this, scatternet alar is very similar to self-expandable, topological deterioration and displacement of devices and very similar to self-healing mesh-mesh. However, the sources examined show that the scatternet field in smart phones distinguishes the user interaction from the braid because of its usage in the installation phase. In our study, scatternet installation, user interaction, taking into account the actual interaction can be applied to a real platform is sought.

\section{III . FOR METHODS FOR MULTI-TABLE MESSAGE}

Scatternet domain is composed of Piconet network with more than one year topology. In this study, using the Bluetree protocol is the logical structure of scatternet. In this study, it is aimed to obtain the scatternet with the widest coverage possible

In the context of my study, studies have shown that when Bluetooth technology is considered a protocol stack - protocol stack for establishing a multi - tabbed communication channel between smart-phones; The sub-level activities such as the 
creation, updating and management of the routing tables should be transferred to the implementation layer. In order to have any data exchange, the Bluetooth connection must be installed with the necessary user approvals. Then, it is possible to transmit the algorithm developed. All process steps, including setting up the Bluetooth connections and creating the scatternet network, are listed below in relation to the topology and device numbers in Figure 1. In the literature, the roles assumed by the devices in the establishment of piconet and scatternet alarn are called master server (S) and slave - client (12).

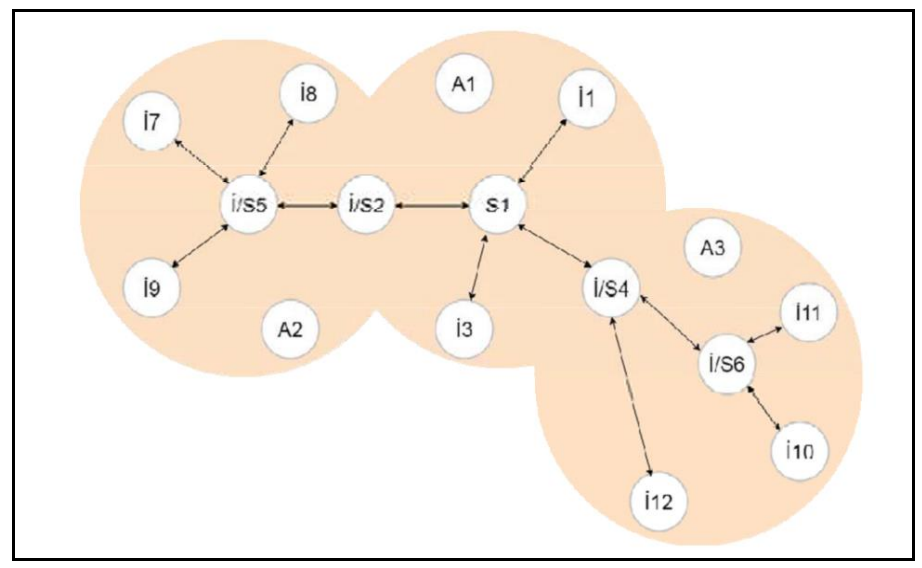

Figure 1. The established scatternet topology.

1. The devices in the middle of the unit must be fitted. The approval of the user must be approved for the assignment of the defect duration. In this case, the devices cannot receive information about the devices they are in between.

2. One device takes on the role of server. (S1)

3. The $\mathrm{S} 1$ device scans the other devices in the media and starts a Thread for each of the strings (A1, 1, / S2, 3, / S4). The devices that are deposited here are filtered through a common service identification number and the said devices are eliminated.

4. A Bluetooth Server Socket object is created on each Thread in S1, and the following connection requests are expected.

5. Candidates in the environment are present. (A1, 1, / S2, 3, / S4)

6. The candidate devices for screening must be connected to the server they have found by creating a Bluetooth Socket object (1, / S2, 3, / S4).

7. When connected to $\mathrm{S} 1,1, / \mathrm{S} 2,3$ and / S4, the device S1 must connect the devices connected to each new connection to the past. If the device is connected to the new device, it should provide the information of the devices that it has been connected to. Thus, it is ensured that every device on the network is aware of the piconeting commons. (Proactive approach)

8. The S1 device manages up to 7 devices connected to it.

9. If a new device is included, one of the hands also activates the server. (/ S2)

10. The I / S2 expects the new connections to be made by creating threaded and Bluetooth Server Socket objects in the background by exploring more remote devices that are recyclable.

11. When a new e device is connected to the I/ S2 device (/ S5), the unit's transmitter transmits the data using Bluetooth Server Socket objects over all the devices (S1) that are attached to it, except the newly connected device.

12. The I / S5 device must transmit to the newly connected e-devices $(7,8,9)$ the list of the contents of the list contained therein.

At the end of the applied steps, scatternet is obtained. The steps covered by the upper steps can be expanded and the network coverage can be expanded. When a message is sent from device $\mathrm{I7}$ to I10, the I7 device uses the Depth First Search (DFS) algorithm to retrieve information that the I / S5 device is the first device to contact I10 to access the $\mathrm{I} 10$ device. The route finding task is also transferred by transferring the message to the I / S5 device.

In this study, one of the advantages of the proposed proactive routing protocol, the alternative DSR and AODV protocols, was that we had to repeatedly broadcast broadcasting to drive the routing path. Scatternet can communicate between them in real time without losing any time after network. The 
only time that is spent is the physical time of the actual data packet. This results in a positive result in terms of real life applications.

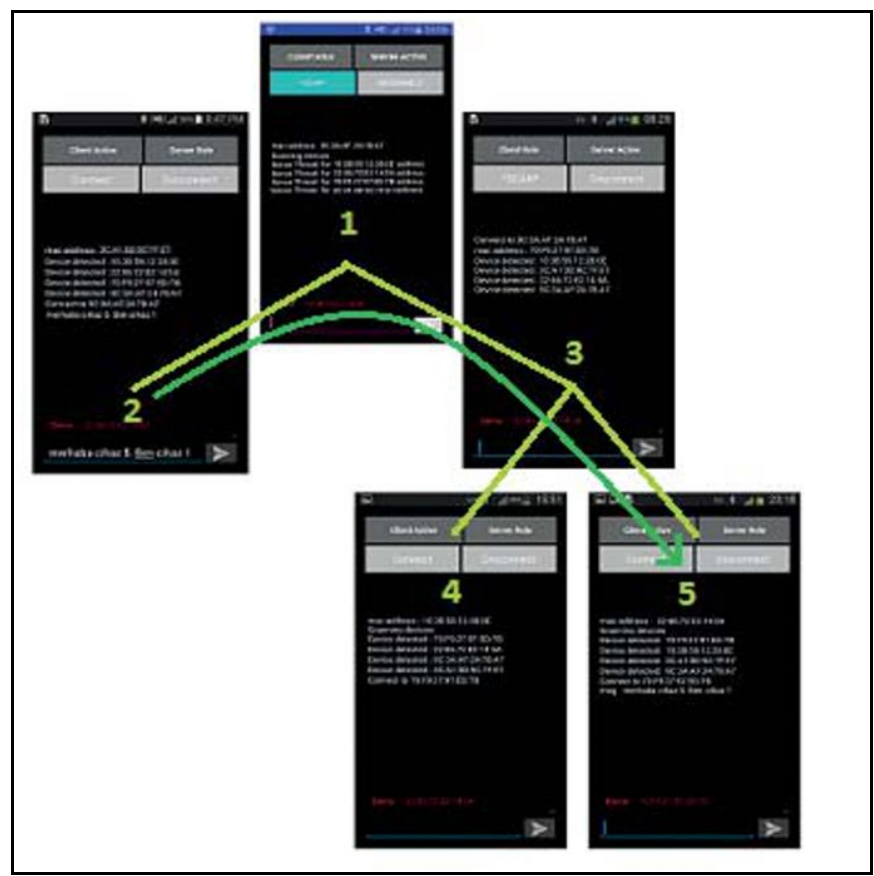

Figure 2. Sending messages on Scatternet 2 devices in real time with multiple tabs

\section{A. Device Discovery}

To perform the simplest communication in Bluetooth technology, the Bluetooth interfaces of the devices must first be activated. It is not only necessary to be active, but also the devices must be recyclable. The ability to find recoveries in the environment with the MAC address and device name of the device. The devices that will assume the role of the consumer should be in the position of being recovered. Using the Bluetooth API, the device's configurability status can be adjusted by passing the time parameter without leaving the application being developed. In operation, independent threads are running to run in the background for all devices that have been scanned in the server role by scanning the media in which the device was found. These threads start the Bluetooth Server Socket independently of the main Thread, which enables screen interaction. Another name is the Thread. Each Thread waits for accepting links to the MAC addresses that are shared.

\section{B. Connection Setup}

The device in the server role starts the corresponding Bluetooth server sockets. The consumer is detectable in the environment of the device. In operation, the names of mobile devices can be assigned via the programmable interface. Up to 7 consumer devices can be connected to a server device as a kst of Bluetooth technology. In other words, piconet consists of up to 8 devices [13].

The client sends a request to connect to one of the servers. The server devices are spreading the available connections. This prevents clients from being routed to a server that cannot be connected. When the client sends the connection request to the server, if I have already performed an interlacing operation between the two devices, the Bluetooth API interrupts at this point and presents a confirmation confirmation message. The client must confirm the message. Since this confirmation message cannot be prevented by overcoming Android's security mechanisms, the network to be installed will have the interaction and interaction of the end-users.

After the connection setup has been performed, both parties are adding the information of the connected device to the globally named connection peers list. Furthermore, user messages between two devices can be sent on both sides.

\section{Application Layer Routing Processes and Message Structure}

When the installed piconet is connected to 3 or higher, messages must be transmitted in a single tab. This requires routing and design of the transmitted message structure. An important task is on the central server device to ensure that all devices on Piconet are aware of each other's presence. In the study, the identification information of each connected to the server is transmitted to the hand by other honey multicasting. This information is stored on the connected peers list of the devices. Thus, all devices on the network can be stored with one or more tabs, which can be accessed by the 
information provided by a network. Scatternet routing process is based on OSI layers. In order to carry out the routing operations in the application layer, a message message must be designed in this layer to convey the data we want to fill. The designed message supports the unicast, multicast and broadcast broadcast types.

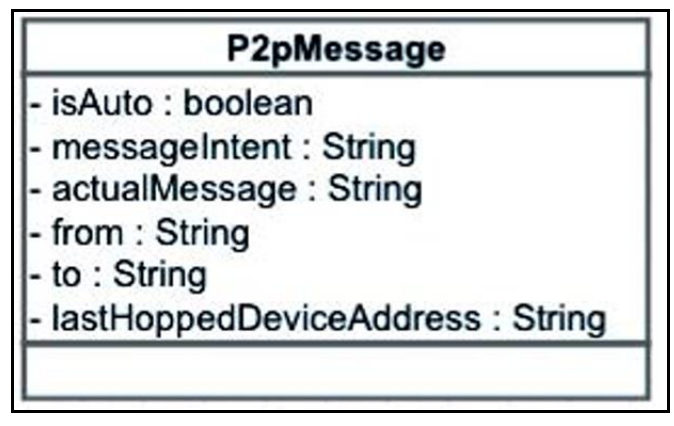

Figure 3. P2pMessage object.

This Auto property in the designed object is used to mark the control messages that are generated as a result of various events on the application. In messages generated by users, this variable is assigned to false. Message Intent refers to the purpose of the original message in the message. The Message Intent value refers to what kind of operations the destination and stop devices will perform on that message. Since the routing process is carried out in the application layer, it also refers to what kind of routing and message propagation method will be used in this layer. message Intent can carry the values of er multicast "or" broadcast abil. The actual Message value is the payload that the message object is carrying. from the source, the message is on the road; to the target device. The last hopped device address value is updated by assigning its own address to the device through which it is forwarded.

The transmission of the P2pMessage object $\mathrm{a}$ is carried out by converting it to the Json text format. It is converted back to the Java object on the Urad mek eler and transmitted back to the Json text format. The Json format is preferred because of the simple and low cost conversion processes and the size of the textual data transferred during the data transmission.

\section{IV.PRODUCTION ANALYSIS AND EVALUATION}

The developed application has been developed by developing a test method to detect delays in message transmission. According to the design, the message generating device must include the date of creation of the message object in the accuracy of the microsecond. When the message reaches the target device, there is a time delay between the two devices, taking the difference between the creation time and the actual time. The behavior of the endto-end delay time is observed by performing a separate calculation on all devices in the module. To ensure that all devices have the same system clock, the device clocks must be synchronized using a Network Time Protocol (NTP) server. The clock from the NTP must be assigned a system administrator role on Android so that the clock can be assigned to the transmission system clock. Applying the root process to mobile devices is undesirable in this study.

To synchronize the time on the devices, the American National Institute of Standards and Technology (NIST) time servers are drawn to the method of withdrawing the time with the HTTP request. Thus, each device in the test environment uses nist.time.gov/actualtime.cgi to calculate the delay on the scatternet by obtaining the time microsecond precision.

$$
g_{\text {toplam }}=\sum_{i=2}^{n}\left(g_{\text {işlem }_{i}}+g_{\text {iletim }_{i}}+g_{\text {yayıllım }_{i}}\right)
$$

Piconet calculates the total delay of at least two devices, in (1), the value $i$ is from 2 . The delay time is indicated by g. Actual time information for all devices is obtained via a. The total delay is calculated by collecting the transmission time of the message and the duration of the transmission of the message between the two devices in order to obtain the time of operation, the duration of the transmission. 
The preferred time server returns the time delay by correcting the delay. For time learning, there is no need to calculate the lost time of the island. In the case where all devices are arranged in a linear manner with a distance of $10 \mathrm{~m}$, the message transmission delay on the scatternet is as shown in Figure 3.

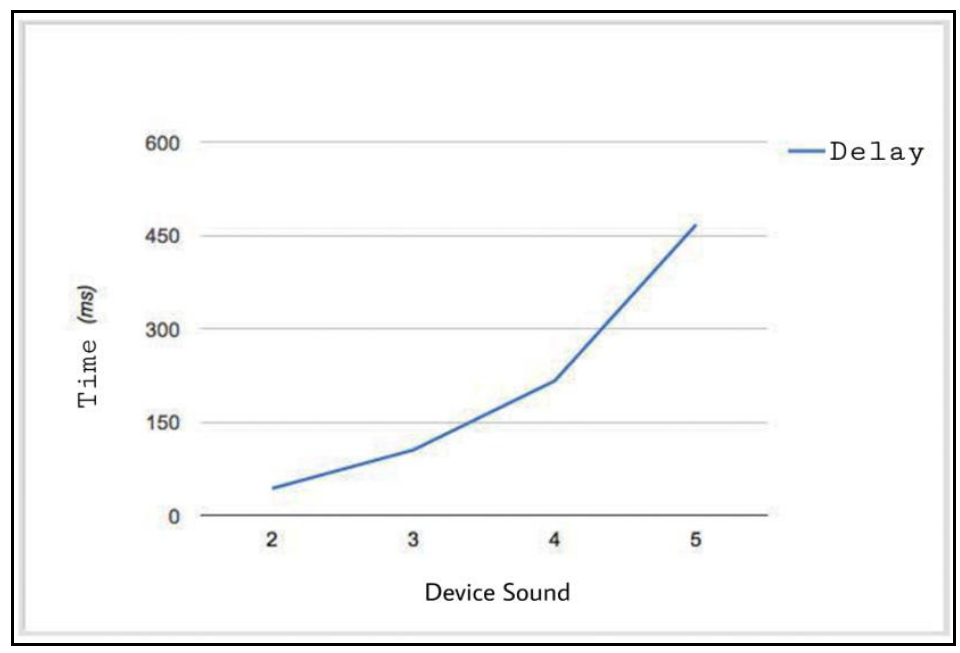

Figure. 4. Delay graph with $10 \mathrm{~m}$ in linear topology.

The distance between devices is fixed. The delay time is calculated taking into account the messaging between the two most remote devices. As it is seen in the graph, as the device counts, each device needs to know and the number of devices is growing. Growing topology is also kept up-to-date on every device at the same time as a network, it also makes it difficult to find the information of the device sought.

Another delay analysis was carried out at an open air distance of approximately $90 \mathrm{~m}$ between devices. As seen in Figure 4, a message sent between 2 devices was transmitted in $1966 \mathrm{~ms}$, making 3 tabs from a distance of about $360 \mathrm{~m}$.



Figure 5. Delay chart with $90 \mathrm{~m}$ intervals in linear topology.

The developed application makes it possible to have a multi-tabbed communication between two smart phones at a distance of approximately 350$400 \mathrm{~m}$. The distance was increased with a similar increase in the delay. Since the work was done in a real platform with real smart phones and not in any simulation environment, an alternative communication method was reached at the development point as it was tested in the field. The configured algorithm, device management, connection installation and routing have been carried out successfully.

The use of reactive protocols such as AODV and DSR, which are recommended in mobile literature, are not suitable for a scatternet network that can be installed with smart-phones. Because the experiments carried out within the scope of this study show that the two smart phones at remote locations are not even aware of the existence of each other, it is not possible to help the pedestrians with the help of coma devices. A phone must first be aware of its existence to try to access the other phone. This situation leads us to ensure that all the devices on the network are aware of each other, primarily during the creation of the network. This protocol allows the network to communicate with each other on the telephone, so that all phones on the network are aware of each other. 
The traditional Blue tree algorithm was preferred at the point of scatternet creation. Hierarchically, one of the smart phones undertakes the role of manager and makes the devices around him. This process continues until the network reaches its limits. On the other hand, a protocol that connects to the network in more than one network topology can be implemented by implementing the scatternet network. However, considering field tests, it will be quite difficult to direct the user and take action. There are methods to make it harder for users to use.

It is not possible to show common performance indicators with other studies since it is not met by a wide-ranging real-life practice, ranging from the development of Android phones to routing protocol design and the maintenance of routing paths. Field experiments of the developed application were found satisfactory in terms of the results obtained. Creating a scatternet network with Android devices directs us to develop a proactive routing protocol. This ensures that, according to the reactive routing protocols, the steering path is much more advantageous in terms of the time of detection.

\section{Results}

In real-life scenarios, where natural disasters and terrorist incidents have rendered the substructure unusable; Nowadays, it is of great importance to develop an alternative communication method that can meet basic needs by using smart-phones. In this work, a multi-tabbed wireless network is installed on the Android platform by selecting Bluetooth technology. The success of the built architecture has been proven by developing an Android based messaging application running on installed network. The Mobile Tasarsz A generation and island packet routing approaches in the literature have been shown to be insufficiently appropriate and effective for scatts installed with smart phones. It is observed that the protocol we proposed in our study presented a holistic solution for the whole process.

It is planned to work on future integration by identifying a tabulation mechanism that will not interfere with the performance and the transmission of live data and video data. With the expansion of the Bluetooth 5 technology in the near future, the current Bluetooth and Bluetooth LE technologies will be available. Nowadays, our approach to building weaves using classic Bluetooth will be supported internally with the Bluetooth 5 protocol. With the experience of Bluetooth 5, we will be able to develop application architectures that are much more effective in the applications of Internet of Things.

\section{References}

[1] S. Zurbes, "Considerations on link and system throughput of Bluetooth networks," in Proc. 11th IEEE Int. Symp. Personal Indoor Mobile Radio Communications, vol. 2, 2000, pp. 1315-1319.

[2] L. Har-Shal, R. Kofneni, A.Segall, and G. Zussman, "Load-adaptive inter-piconet scheduling in small-scale sbuetooth scatternets," IEEE Communications Magazine, pp. 136-142, July 2004

[3] E.B. Gabrielsen, "An API to Wi-Fi Direct using reactive building blocks," Norwegian University of Science and Technology Department of Telematics, June 2012

[4] Wi-Fi Peer-to-Peer (P2P) Technical Specification Version 1.7

[5] V. Rönnholm, "Push-to-Talk over Bluetooth," 39th Hawaii International Conference on Science, 2006

[6] C.Y Chang, P.K. Sahoo, S.C. Lee, "LARP: A novel routing protocol for the Bluetooth," Wireless and Optical Communications Networks, 2005

[7] Y.Wang, I,Stojmenovic, and X.Y.Li, "Bluetooth scatternet formation for singlehop ad-hoc networks based on virtual positions," Computers and Communications, 2004 
[8] T. Salonidis, P. Bhagwat, and L. Tassiulas, R. LaMaire, "Distributed topology construction of Bluetooth personal area networks," INFOCOM'01, Anchorage, Alaska, pp. 1577-1586, April 2001.

[9] C. Pamuk, E. Karasan, "A tree-based energy-efficient distributed A algorithm for forming bluetooth scarnet topologies," MedHoc-Net'04, June 2004.

[10] G. V. Zamba, S. Basagni, and I. Chlamtac, "Bluetrees-scattemet formation to enable Bluetooth based ad-hoc networks," ICC'2001, pp. 273-277, June 2001

[11] C. Petrioli, S. Basagni, 1. Chlamtac, "BlueMesh: degree - constraint multi-hop scanemet formation for bluetooth networks," Mobile Networks and Applications, vol. 9, no. I, pp. 33-47,2004.

[12] B. Donegan, D. Doolan, and S. Tabirca "Mobile Message Passing using a Scatternet Framework," International Journal of Communications \& Control, May 2007, Vol. 3, Issue 1, pp. 51-60

[13] K.V.K.K. Prasad, Principles of Digital Communication Systems and Computer Networks, 2nd ed., vol. 36, 2008, pp.592596

[14] M.Methfessel, S.Peter, and S. Lange, "Bluetooth Scatternet Tree Formation for Wireless Sensor Networks," 8th IEEE International Conference on Mobile AdHoc and Sensor Systems, 2011, pp. 789794

[15] C.M. Yu, Y.B. Yu, “A Variant-Hop Algorithm in Forming Bluetooth Sensor Networks," 8th IEEE International Conference on Networking, Architecture and Storage, 2013, pp. 302-306

[16] C.M. Yu, Y.B. Yu, "Reconfigurable Algorithm for Bluetooth Sensor Networks,"
IEEE Sensors Journal, Oct 2014, Vol. 14, no. 10 , pp. $3506-3507$

[17] C. Jung, K. Kim, J. Seo, B.N. Silva, and K. Han, "Topology Configuration and Multihop Routing Protocol for Bluetooth Low Energy Networks", IEEE Access, vol. 5, 2017, pp. 9587-9598

[18] Z. Guo, I.G. Harris, T.F. Tsaur, and X. Chen, "An On-demand Scatternet Formation and Multi-hop Routing Protocol for BLE-based Wireless Sensor Networks," IEEE Wireless Communications and Networking Conference (WCNC), 2015, pp. 1590-1595 